\title{
A BIG HEALTH APPLICATION USED IN SMART CLOTH \& GLASS
}

\author{
Athira Sudersanan \\ Final Year BTech CSE \\ Sree Buddha College of Engineering \\ Pattoor,Kerala,India
}

\author{
Alina Shaji \\ Final Year BTech CSE \\ Sree Buddha College of Engineering \\ Pattoor,Kerala,India
}

\author{
Dhanya Sreedharan \\ Asst.Professor,CSE \\ Sree Buddha College of Engineering \\ Pattoor,Kerala,India
}

\begin{abstract}
Nowadays, the people to face problems, such as not regular sharing of medical resources, the spreading chronic diseases, dietary issues and the growing medical overheads. Combining the current information technology into the healthcare system will largely mitigate the issues. This paper presents the big health application system based on the health Internet of Things and big data.
\end{abstract}

Keywords: ; Big health, wearable computing, cloud computing, Internet of Things, big data, Dietary monitoring.

\section{INTRODUCTION .}

Big health is a challenging industry, which is defined by people in centre, maintaining person's lifecycle from birth to death, from hindering to restoring and including industry from government to market. The majority of big health includes health product market, health service market, real estate market and finance department. Humans sophisticated from diseases of which $25 \%$ would be fully hindered, $25 \%$ would be investigated in the beginning; and the remaining could be done with belligerent treatment to improve quality of life in a high scale. By building up early detection mechanism, majority of diseases could be controlled. Every human might have experienced a procedure of health to disease atleast once in their lifecycle. [1]
In order to merge the primary medical services and to improve primary healthcare quality, bighealth could use mobile medical health system, bigdata, wearable device, a new generation of mobile communication technology. It also control the growth rate of a variety of critical and persistent diseases, increase people's realization of health management and disease prevention.

Nowadays , the people's dietary habit is reordering because of their busy life style .People being prone to bounce their meals or they eat unevenly. People turn to food for condolence and consolation. In addition to this messy eating ,it shortens the meal time.

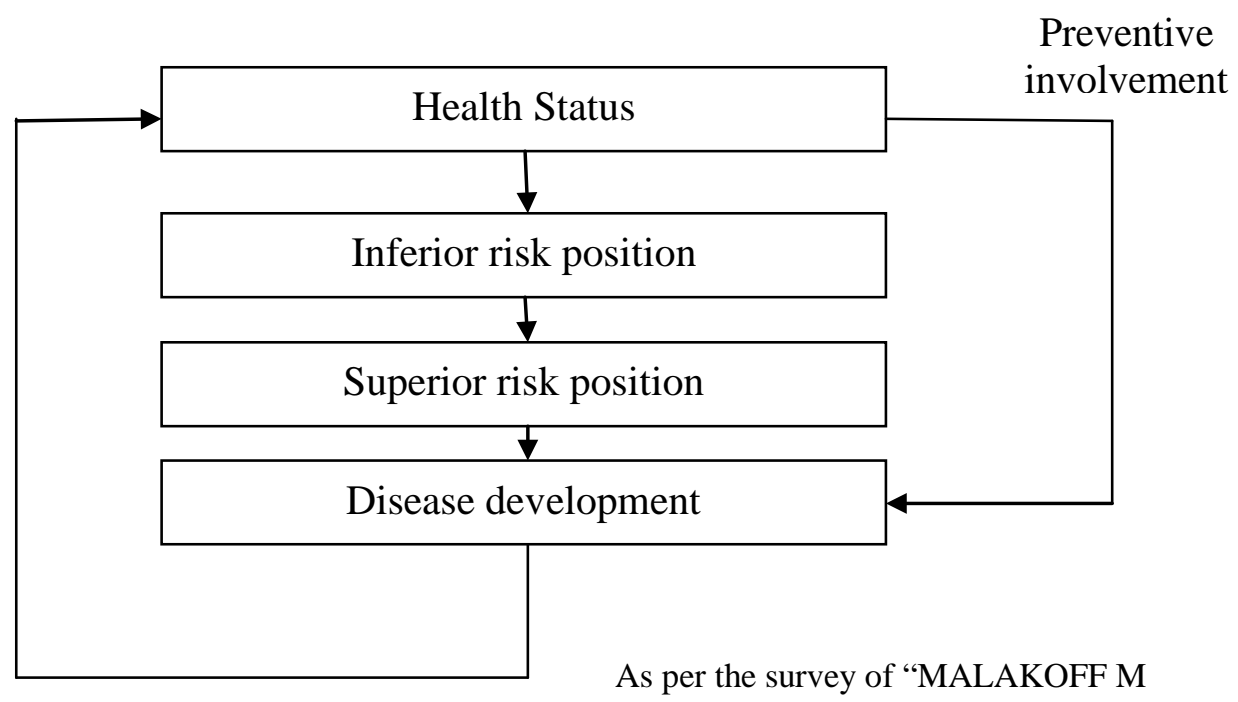

Prevention,retard and Reverse of diseases

Normally the ranking of health is trom int to superior risk position , position to the occurrence of early bruise position to clinical indication and finally to disease
Fig 1: Health Representation

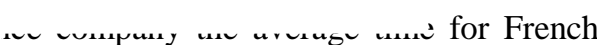
ch had declined from 90 mts 20 years ago to 22 minutes. People rush through meal with not enough proper chewing of food rigorously. This may lead to 
overweight , risk of developing cancer, cardiovascular disease and diabetes[2]. Dietary monitoring can provide private and comprehensive information of disease features ( intake schedule ,meal composition, food category)to prevent and intervene with unhealthy dietary habits. It provides valuable information for chronic disease diagnosis. Dietary logging is a built in function in commercial wearable devices and some Smartphone apps[3].

Dietary can be monitored daily with high accuracy by integrating electromyography sensors into arm of eye glasses. It allows monitoring in comfortable manner. Most of the people wear glasses according to the survey of vision council of America, 64\% of adults wear glasses. More than traditional glasses, smart glasses are being admired by many people. Therefore glasses are more suitable for dietary monitoring. It is discovered that when people wear glass, it touches lower part of temporalis muscle. Temporalis is a known mastication muscle whose function is to support chewing process. While clenching the teeth, the temporalis begin to contract which specify the possibility of using EMG sensors to monitor mastication and there by monitor dietary related behavior. In comparison with existing solutions(video or audio recording) the proposed solution of EMG sensors will cause less privacy violation[4-5].

\section{ARCHITECTURE}

This paper is supported by health IoT and Big data, which represents cloud to-end fusion, big health application system architecture. The architecture consists of

- BIG HEALTH PERCEPTION LAYER

- TRANSPORT LAYER OF BIG HEALTH SYSTEM

- HEALTH CLOUD SERVICE LAYER

- CLOUD TO END FUSION complex signal accession framework, we need multiple sensor nodes working together. These nodes enable cooperative communication signal that require wireless communication technology in perception layer[6-8].

\section{B. Transport Layer of Big Health}

It sends control instructions of upper application to sensor node and receive data acquired by perception layer. The provocation faced by transport layer include (1) consolidation of existing network and perception layer network is a big challenge. (2) The scope of health IoT is extensively ,covers not only large scale IoT but also covers small scale IoT. For our purposes we can use equipments like relay module, communication networks. These devices accept data, and after processing ,transfer it to upper layer[9-10].

\section{Health Cloud Service Layer}

It can be divided into two sub-layers:

- .BIG HEALTH CLOUD SERVICE SUPPORT SUBLAYER

- BIG HEALTH CLOUD SERVICE APPLICATION SUB-LAYER

The main task of cloud service support sub- layer is to collect observational data, then you can simultaneously acquire physiological data such as EEG, ECG, EMG, blood pressure with an external data acquisition system. And then these data it. Compared to other layers, this layers, this layer should be squeezed, keep, prepare to receive and examine has vital role in health services and applications. methodically in detail, typically inorder to explain and interpret.

Health cloud service application sub-layer is accountable for check all sensor nodes, visual presentation of data, the scheme of workflow and business. In health IoT, big health service

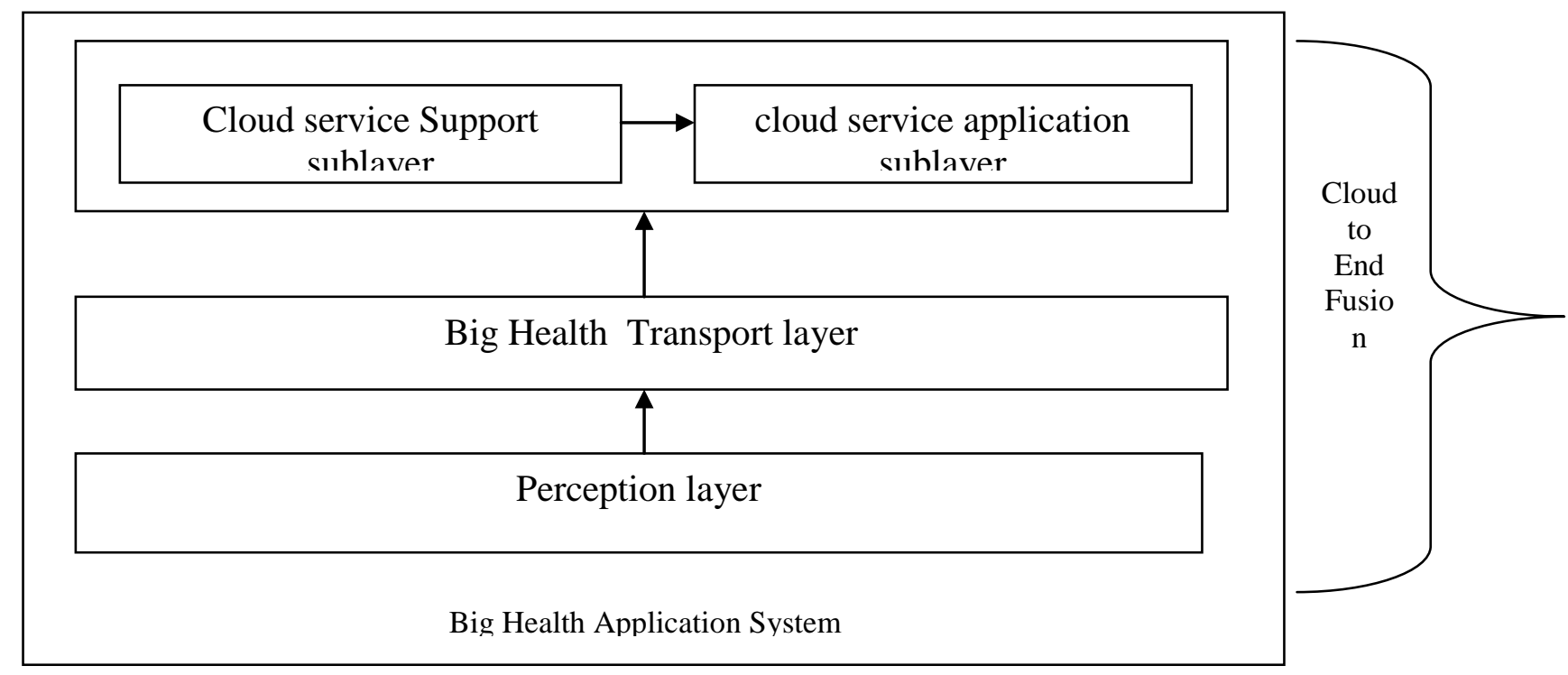

Fig 2 : Architecture

\section{A. Big Health Perceoption Layer}

Big health perception layer has two aims: (1)Its main components are different types of sensors which obtain the original signal of the world. (2) Collecting different physiological signal associated with human health. In many cloud application sub layer is also a layer of cloud to end fusion.

\section{Cloud to End Fusion}

Cloud to end fusion has very long far beyond traditional scope 
of IoT. Cloud robotics is a field of robotics that attempts to invoke cloud technologies such as cloud computing, cloud storage and other internet technologies centered on the benefits of converged infrastructure and shared services for robotics. When connected to cloud, robots can benefit from the powerful computation, storage and communication resource of modern data center in the cloud, which can process and share information from various robots or agent(other machines, smart objects, humans etc). Humans can also delegate tasks to robots remotely through networks. Thus it is possible to build lightweight, low cost; smarter robots have intelligent "brain" in the cloud. The brain consists of data center, knowledge base, task planners, communication support etc[11].

\section{KEY TECHNOLOGIES}

\section{A. Wearable Devices}

Wearable devices and the data generated by them gives a unique opportunity to understand the user behavior, health etc. Now a days, lot of data is generated by the wearable devices. This data is highly unique due to personal nature of wearable devices. There is an exponential growth in wearable devices and they collect a lot of data which cannot be processed using conventional techniques. we will introduce smart cloth for collecting physiological signal to help depressive patients, autism children, driver, aquanaut pilot, empty-nest elderly people and glass to monitor diet[12].

\section{B. Mobile Communication Technology}

Big health system is very applicable in areas like urban ,rural, mountainous areas etc .Today, mobile healthcare has big role. The wireless communication technologies used are:

Low power, low data rate,v Bluetooth Low Energy (BLE) $v$ Wireless Technologies for Wearables Several technologies are being used today in wearables, with Bluetooth being the most prevalent. < GPS for positioning Mobile network (GSM) v Low power, low data rate, short range $(20 \mathrm{~cm}$.) v Near Field Communications (NFC) v Medium power, high data rate,100 meter range. $v$ Wireless LAN (WiFi) v100 meter range[13].

\section{Cognitive Computing}

The main human health problem are physical, spiritual and mental with the rapid development of wearable devices. All are now focused on mental health, which is the trend of health IoT. By the rapid development of wearable device, we can make emotional interaction feedback with the user. Wearable devices perform many functions about physical information, location information, and social networking information of human. But there exist one disadvantage, by analyzing human emotion, we

cannot focus on spiritual care[14].

\section{Health Care Cloud Robot}

Today, robotics industry is in the developing stage. Healthcare robot has influence in many fields like artificial intelligence, (c) 2015-19, IJARCS All Rights Reserved machinery and electronics, intelligent voice recognition, mobile multimedia communications, sensor networks, telemedicine etc. The application of scientific knowledge for practical purposes, especially in industry of early robotics are automation and computer technology. It amalgamate various details of technologies, which is used in industrial control field[15].

\section{E. Health Cloud \& Big Health Data}

Health big data based on mobile cloud includes following features
- Heterogenity
-High correlation
-Real-time
- Time and space correlation
-Low proportion of valid data

\section{TYPICAL APPLICATIONS}

\section{A. Sustainable Healthcare Based on Smart Clothing}

In this, it encapsulate a variety of physiological indicators and has benefits like cosy, worthy for elderly people, and the people with chronically ill and also people with mental illness. Chronically ill means a situation where a person is unable to perform atleast two activities of daily living such as eating, bathing, toileting and dressing, In this first we collect bioelectrical signal of the human body. Dry textile electrode is used to measure and monitor these signals. On amalgamate it into fixing, the psychological feeling of human is identified. The dry textile electrode has many features like soft, washable, breathable, does not feel the presence .Users simply wear , it would monitor electrocardiography signal, respiration signal, heart rate and all other signals in human body. This is very helpful to identify diseases and to diagnose it. Smart clothing based healthcare application scenario are depressive patients, autism children, driver, aquanaut pilot, empty-nest elderly people. By wearing the smart cloth physiological datas like ECG, SpO2, blood pressure, blood glucose, respiration, body temperature, heartbeat rate etc can be recognized. Then Health data processing, analysis and prediction based on Big data is performed. Massive data processing framework includes batch processing, streaming processing. Various algorithms used are Machine learning, deep learning algorithm, Mahout. If a service robot is used for emotion interaction application framework, it is very useful. Emotional interaction plays major rule in whole application framework. The robot gives best emotional support interaction. The favours of emotional robots interaction include music, video, dance, voice synthesis etc in fig 3. 


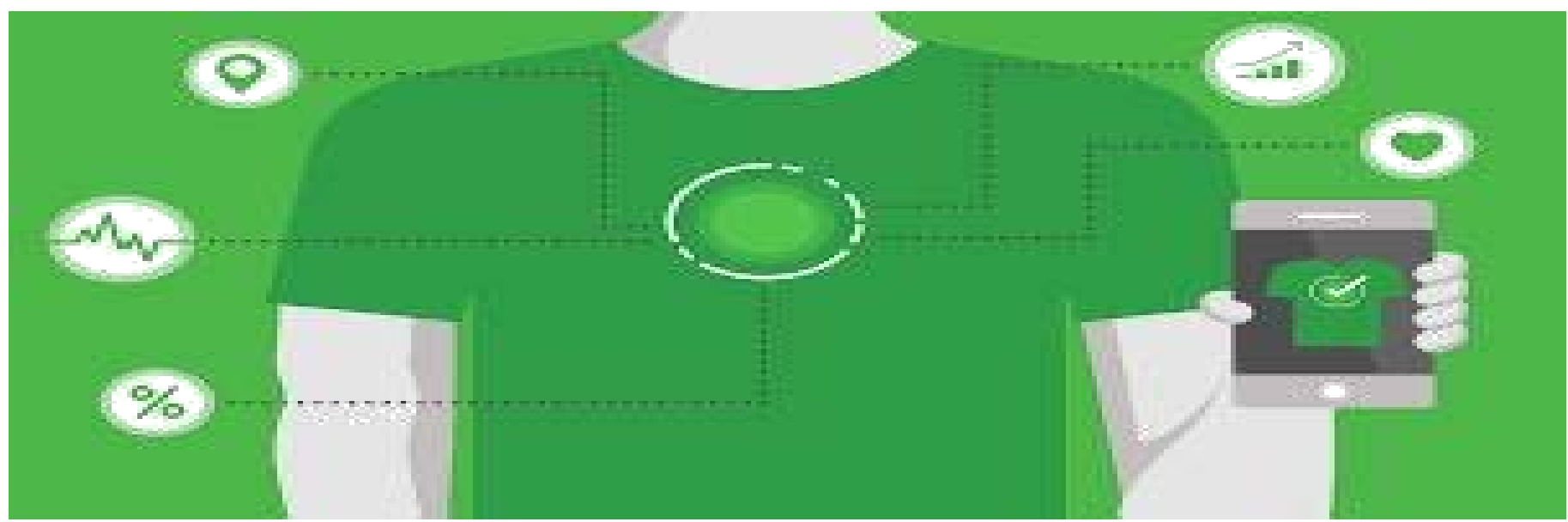

Fig 3: Healthcare system architecture based on smart clothing

\section{B. Dietary Monitoring using Electromyography Sensors}

There are mainly three components : diet aware glasses , central processor, health data cloud in fig 4. The central processor is accountable for data processing and provide feedback to the user. Glasses are in touch with central processor via Bluetooth. User's dietary information is uploaded to health data cloud by central processor. The health data contains user's exercise and sleep record. Thereby cloud provide user with detailed and individualized dietary guidance.

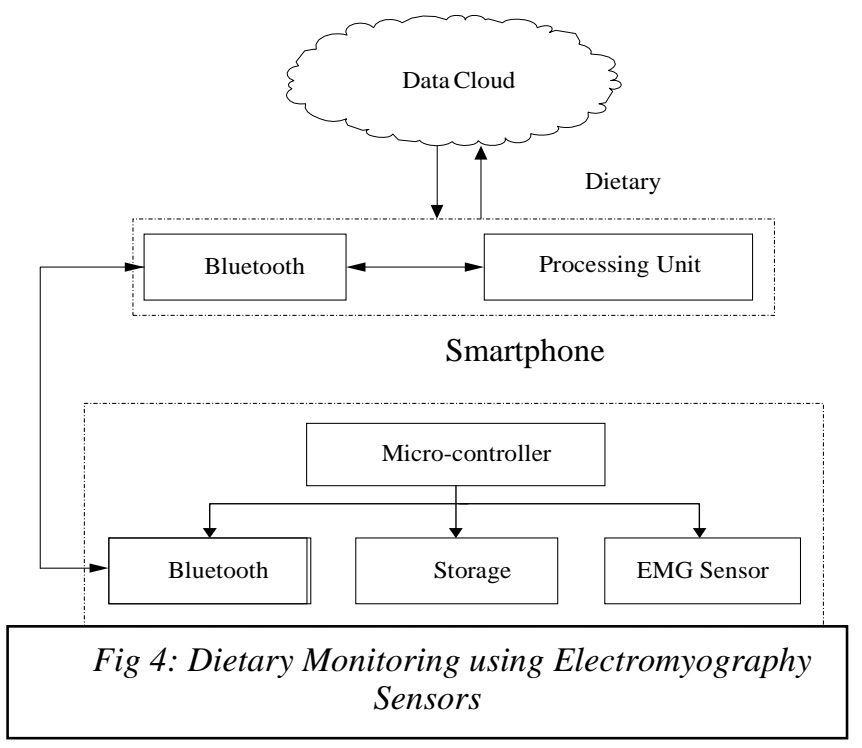

It involves an EMG sensor , a micro- controller , a Bluetooth radio an SD breakout shield and an SD card mounted on the glasses. The glasses have two modes ;idle mode and working mode. By revert the glasses are in idle mode.EMG signals are sampled at certain frequency .Based on sensor data glasses determine whether user is probably eating or not. The conclusion is made by comparing signal variance to adaptive threshold. The contemptuous data frames are tossed out. The glasses switches to working mode if it diagnose that the user is eating something. In working mode ,Emg sensors
Chewing detection :

It would sample at high frequency and transmit data to central processor for fine grained data processing. If Smartphone is not nearby then it would temporarily store data to on-board SD card.

Chewing is the rudimentary process during food intake. It provides fine-grained information on number of chewing cycles and review intake schedule. Chewing detection algorithm is divided into two parts on board real time and coarse grained decision . Food classification:/meal composition is a prior criteria for dietary monitoring, which supports chronic prevention and diagnosis.

\section{CONCLUSION}

The current information technologies may be used into healthcare to affected global health issues such as not regular sharing of medical resources, the spreading chronic diseases, dietary issues and the growing medical overheads. In this paper, we introduce a big health application system which is based on the health IoT applications and big data. It suggest the big health care system's architecture, technology challenges and several typical large health applications.

\section{REFERENCES}

[1] Y.-J. Ma, Y. Zhang, O. M. Dung, R. Li, and D.-Q. Zhang, "Health Internet of Things: Recent applications and outlook," J. Internet Technol., vol. 16, no. 2, pp. 351_362, 2015.

[2] O. Amft, M. St"ager, P. Lukowicz, and G. Tr"oster, "Analysis of chewing sounds for dietary monitoring," in UbiComp, Tokyo, Japan, 2005, pp. 56-72

[3] O. Amft and G. Tr"oster, "On-body sensing solutions for automaticdietary monitoring," IEEE Pervasive Computing, vol. 8, no. 2, pp. 62- 70, 2009.

[4] K.-h. Chang, S.-y. Liu, H.-h. Chu, J. Y.-j. Hsu, C. Chen, T.-y. Lin, C.-y. Chen, and P. Huang, "The diet-aware dining table: Observing dietary behaviors over a tabletop surface," in Pervasive, Dublin, Ireland, 2006, pp. 366-382.

[5] J. Cheng, O. Amft, and P. Lukowicz, "Active capacitive sensing: Exploring a new wearable sensing modality for 
activity recognition,” in Pervasive, Helsinki, Finland, 2010, pp. 319-336.

[6] G. Aloi et al., "'Software de_ned radar: Synchronization issues and practical implementation," in Proc. Int. Conf. Cognit. Radio Adv. Spectr. Manage. (CogART), Barcelona, Spain, Oct. 2011, pp. 1_5.

[7] M. Chen, Y. Zhang, L. Hu, T. Taleb, and Z. Sheng, “'Cloudbased wireless network: Virtualized, recon_gurable, smart wireless network to enable 5G technologies," Mobile Netw. Appl., vol. 20, no. 6, pp. 704_712, Dec. 2015.

[8] V. C. Gungor, P. Pace, and E. Natalizio, “AR-TP: An adaptive and responsive transport protocol for wireless mesh networks," in Proc. IEEE Int. Conf. Commun. (ICC), Jun. 2007, pp. 3740_3745.

[9] E. Sakhaee, T. Taleb, A. Jamalipour, N. Kato, and Y. Nemoto, "A novel scheme to reduce control overhead and increase link duration in highly mobile ad hoc networks," in Proc. IEEE Wireless Commun. Netw. Conf., Mar. 2007, pp. 3972_3977.

[10] S. Yessad, F. Nait-Abdesselam, T. Taleb, and B. Bensaou, “'RMAC: Reservation medium access control protocol for wireless sensor networks," in Proc. 32nd IEEE Conf. Local Comput. Netw. (LCN), Oct. 2007, pp. 719_724.

[11] Y. Ma, Y. Zhang, J. Wan, D. Zhang, and N. Pan, “'Robot and cloud assisted multi-modal healthcare system," Cluster Comput., vol. 18, no. 3, pp. 1295_1306, 2015.

[12] M. Chen, S. Gonzalez, A. Vasilakos, H. Cao, and V. C. M Leung, "Body area networks: A survey," Mobile Netw. Appl., vol. 16, no. 2, pp. 171_193, 2011.

[13] X. Ge, J. Ye, Y. Yang, and Q. Li, “'User mobility evaluation for 5G small cell networks based on individual mobility model," IEEE J. Sel. Areas Commun., vol. 34, no. 3, pp. 528_541, Mar. 2016.

[14] I. S. Jacobs and C. P. Bean, "Fine particles, thin films and exchange anisotropy,” in Magnetism, vol. III, G. T. Rado and H. Suhl, Eds. New York: Academic, 1963, pp. 271-350.

[15] M. Chen, Y. Ma, S. Ullah, W. Cai, and E. Song, "'ROCHAS: Robotics and cloud-assisted healthcare system for empty nester," in Proc. Int. Conf. Body Area Netw., 2013, pp. 217220. 